\title{
A Feasibility Study on a Monitoring for Seagrass beds Using Environmental DNA
}

\author{
Maiko Akatsuka $\ddagger$ \\ ‡ Taisei corporation, Yokohama, Kanagawa, Japan
}

Corresponding author: Maiko Akatsuka (aktmik00@pub.taisei.co.jp)

Received: 01 Mar 2021 | Published: 04 Mar 2021

Citation: Akatsuka M (2021) A Feasibility Study on a Monitoring for Seagrass beds Using Environmental DNA. ARPHA Conference Abstracts 4: e65262. https://doi.org/10.3897/aca.4.e65262

\begin{abstract}
In order to protect marine resources listed in the SDGs, it is important to conduct surveys to understand the current status and transition of aquatic species such as fish and seagrass. However, the area and frequency of surveys are often limited due to the lack of manpower and cost. Environmental DNA (eDNA) analysis is a method to obtain information of aquatic species in the sea or rivers. The aquatic species are identified by analyzing DNA contained in the sampled water. The eDNA analysis can be utilized as a new and efficient method for investigating aquatic species.
\end{abstract}

The author is developing a monitoring method for seagrass beds using eDNA.

Seagrasses are sessile organisms and don't move like a fish, the areas where eDNA is generated are fixed. Therefore, eDNA path in a seagrass beds area can be predicted by flow prediction simulation. The monitoring carries out in the path of eDNA released from seagrass.

When there is a correlation between the amount of DNA and the amount of biomass, it is possible to gain knowledge of the amount of biomass from sampling water in the path of eDNA. For example, the changes in the amount of biomass due to growth and withering of seagrass beds can be studied by observing the changes in the amount of DNA.

The purpose of this study is to examine the feasibility of the monitoring method using experiments, field surveys and numerical simulations for eDNA. 
The water tank experiments for 15 months, suggested that the amount of seagrass eDNA is related to the seasonal changes in the biomass of seagrass, which has been grown in the water tank. In the conducted four field surveys, the amount of eDNA tended to be high during times of spring with high amounts of seagrasses. In addition, the decomposition process of eDNA were examined by laboratory experiments using sea water. As a result, seagrass DNA contained in the sampling sea water was degraded and undetected within around 5 days. This result suggests that information obtained from the sampling water reflects biological information within a few days. These results suggest the possibility to estimate the changes in biomass by using eDNA in the sea.

And, in the field surveys, it was shown that the amount of eDNA is small, so we have recognized that increasing the amount of sampling water and improvement of the DNA analysis method is an issue.

This monitoring method is feasible when the collected eDNA is related to a specific seagrass bed. The feasibility of the method was considered using numerical simulation.

In the numerical simulation, a particle tracking method using 10 types of simple bay shapes was used to trace the path of eDNA regarded as a particle.

When the eDNA started from multiple positions, it was searched for position where the point where DNA starts and arrives is uniquely determined.

As a result, at some observation points, the particles that departed from a specific position was observed without being mixed with particles departing from other starting positions, and this was true for all bay types. In addition, the same tendency could be obtained in calculations using the bathymetry of Ago bay, in Mie prefecture.

These suggests that it may be possible to monitor seagrass beds by performing fixed-point observations according to the seagrass beds distribution and bay flow.

Since few cases in the sea were conducted, the authors intend to continue the survey for the upcoming years and continue the study under various conditions including numerical analysis.

\section{Keywords}

environmental DNA, particle trackng simulation, biological monitering, seagrass, Zostera marina

\section{Presenting author}

Maiko AKATSUKA 


\section{Presented at}

1st DNAQUA International Conference (March 9-11, 2021) 